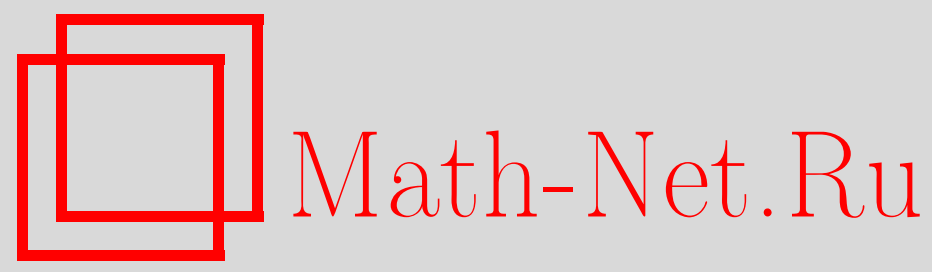

А. Е. Соловьев, Интерполяция с градиентом в шаре и полидиске, Матем. заметки, 1999, том 66, выпуск 3, 407-416

DOI: https://doi.org/10.4213/mzm1182

Использование Общероссийского математического портала Math-Net.Ru подразумевает, что вы прочитали и согласны с пользовательским соглашением http://www . mathnet.ru/rus/agreement

Параметры загрузки:

IP: 52.87 .193 .239

26 апреля 2023 г., 16:02:25

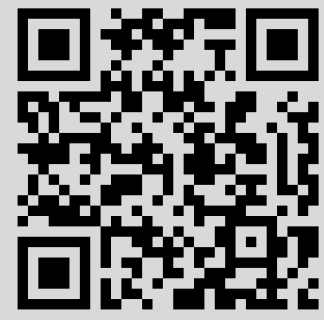




\section{ИНТЕРПОЛЯЦИЯ С ГРАДИЕНТОМ В ШАРЕ И ПОЛИДИСКЕ}

\section{А. Е. Соловьев}

В статье рассматривается задача интерполяции с градиентом для банаховых алгебр $H^{\infty}\left(\mathbb{B}^{n}\right)$ и $H^{\infty}\left(\mathbb{D}^{n}\right)$. Получены достаточные условия разрешимости этой задачи.

Библиография: 4 названия.

1. Введение. Пусть $\mathbb{B}^{n}$ обозначает единичньй шар в пространстве $\mathbb{C}^{n}$

$$
\mathbb{B}^{n}=\left\{z=\left(z^{1}, \ldots, z^{n}\right) \in \mathbb{C}^{n}:|z|^{2}=\left|z^{1}\right|^{2}+\cdots+\left|z^{n}\right|^{2}<1\right\}
$$

a $\mathbb{D}^{n}$ - единичный полидиск в $\mathbb{C}^{n}$

$$
\mathbb{D}^{n}=\left\{z=\left(z^{1}, \ldots, z^{n}\right) \in \mathbb{C}^{n}:\left|z^{1}\right|<1, \ldots,\left|z^{n}\right|<1\right\} .
$$

Обозначим через $\mathcal{D}^{n}$ любую из областей $\mathbb{B}^{n}$ или $\mathbb{D}^{n}$ и рассмотрим банахову алгебру $H^{\infty}\left(\mathcal{D}^{n}\right)$ ограниченных голоморфных функций в $\mathcal{D}^{n}$ с нормой

$$
\|f\|_{H^{\infty}}=\sup _{z \in \mathcal{D}^{n}}|f(z)|, \quad f \in H^{\infty}\left(\mathcal{D}^{n}\right) .
$$

Напомним, что последовательность $\left\{a_{k}\right\}_{k=1}^{\infty}$ точек $\mathcal{D}^{n}$ назьвается интерполяционной для $H^{\infty}\left(\mathcal{D}^{n}\right)$, если для любого ограниченного набора комплексных чисел $\left\{f_{k}\right\}_{k=1}^{\infty}$ найдется функция $F \in H^{\infty}\left(\mathcal{D}^{n}\right)$ такая, что $F\left(a_{k}\right)=f_{k}$.

Обозначим через

$$
\langle z, \bar{w}\rangle=\sum_{i=1}^{n} z^{i} \overline{w^{i}}
$$

эрмитово произведение в $\mathbb{C}^{n}$, через

$$
\rho(z, w)=\left(1-\frac{\left(1-|z|^{2}\right)\left(1-|w|^{2}\right)}{|1-\langle z, \bar{w}\rangle|^{2}}\right)^{1 / 2}
$$

гиперболическую метрику в $\mathbb{C}^{n}$.

В одномерном случае Карлесоном [1] был доказан следующий критерий интерполяционности.

Работа выполнена при частичной финансовой поддержке Российского фонда фундаментальных исследований (гранты № 98-03-00640 и № 96-15-96131). 
Теорема А. Пусть $n=1$. Последовательность точек $\left\{a_{k}\right\}_{k=1}^{\infty}$ круга $\mathcal{D}^{1}=$ $\mathbb{B}^{1}=\mathbb{D}^{1}$ является интерполяционной для $H^{\infty}\left(\mathcal{D}^{1}\right)$ тогда и только тогда, когда существует константа $\delta>0$ такая, что для любого $k$ справедлива оченка

$$
\prod_{j: j \neq k} \rho\left(a_{j}, a_{k}\right) \geqslant \delta
$$

или, что әквивалентно, найдется константа $C>0$ такая, что для любого $k$ имеет место неравенство

$$
\sum_{j: j \neq k}\left(1-\rho\left(a_{j}, a_{k}\right)\right) \leqslant C
$$

При $n>1$ ситуация в корне меняется - возникает “зазор" между необходимыми и достаточньми условиями интерполящионности. Достаточное условие Берндтссона для $H^{\infty}\left(\mathbb{B}^{n}\right)[2]$ выглядит так же, как и у Карлесона.

ТЕоремА В. Пусть существует константа $\delta>0$ такая, что для любого $k$ справедлива оченка

$$
\prod_{j: j \neq k} \rho\left(a_{j}, a_{k}\right) \geqslant \delta
$$

Тогда последовательность $\left\{a_{k} \in \mathbb{B}^{n}\right\}_{k=1}^{\infty}-$ интерполяичонная для $H^{\infty}\left(\mathbb{B}^{n}\right)$.

Похоже вьглядит и достаточное условие Берндтссона для $H^{\infty}\left(\mathbb{D}^{n}\right)$ [3]. Обозначая через

$$
\rho^{s}(z, w)=\left|\frac{z^{s}-w^{s}}{1-\left\langle z^{s}, \overline{w^{s}}\right\rangle}\right|
$$

гиперболическое расстояние между проекциями $z$ и $w$ на $s$-ю координатную плоскость в $\mathbb{C}^{n}$, а через

$$
\rho^{*}(z, w)=\max _{1 \leqslant s \leqslant n}\left(\rho^{s}(z, w)\right)=\max _{1 \leqslant s \leqslant n}\left|\frac{z^{s}-w^{s}}{1-\left\langle z^{s}, \overline{w^{s}}\right\rangle}\right|
$$

поликруговую метрику в $\mathbb{C}^{n}$, можно сформулировать результат Берндтссона следующим образом.

ТЕОрема С. Пусть существует константа $\delta>0$ такая, что для любого $k$ выполняется неравенство

$$
\prod_{j: j \neq k} \rho^{*}\left(a_{j}, a_{k}\right) \geqslant \delta
$$

Тогда последовательность $\left\{a_{k} \in \mathbb{D}^{n}\right\}_{k=1}^{\infty}$ - интерполячионная для $H^{\infty}\left(\mathbb{D}^{n}\right)$.

Однако, необходимое условие, принадлежащее Варопулосу [4], не совпадает с достаточным. А именно, имеет место

ТЕОрема D. Если последовательность $\left\{a_{k} \in \mathbb{B}^{n}\right\}_{k=1}^{\infty}$ - интерполяиионная, то найдется константа $C>0$ такая, что для любого $k$ справедлива оченка

$$
\sum_{j: j \neq k}\left(\frac{\left.\left(1-\left|a_{j}\right|^{2}\right)\left(1-\mid a_{k}\right)^{2}\right)}{\left|1-\left\langle a_{k}, \overline{a_{j}}\right\rangle\right|^{2}}\right)^{n} \leqslant C .
$$


Более того, Берндтссон [2] показал, что степень $n$ в условии Варопулоса, вообще говоря, нельзя уменьшить. Точнее, для любого $\varepsilon>0$ существует интерполяционная последовательность $\left\{a_{k} \in \mathbb{B}^{n}\right\}_{k=1}^{\infty}$ такая, что

$$
\sum_{k}\left(1-\left|a_{k}\right|^{2}\right)^{n-\varepsilon}=\infty
$$

Можно ожидать, что изменив условие интерполящии (потребовав, скажем, чтобы интерполировались не только значения функции в точках $\left\{a_{k}\right\}$, но и направления ееградиента), удастся ликвидировать “зазор” между необходимыми и достаточньми условиями интерполяционности.

Задача интерполяции с градиентом рассматривается в этой работе и для нее получены аналоги теорем В и С.

Далее автор использует следующие обозначения: $\partial_{s}=\partial / \partial z^{s}-$ дифференцирование по $s$-й координате и

$$
\partial_{m} f=\sum_{s=1}^{n} \partial_{s} f \cdot m^{s}
$$

- дифференцирование по направлению вектора $m$.

2. Основные определения и леммы. Для того, чтобы сформулировать задачу интерполяции с градиентом нам понадобятся следующие леммы.

ЛЕМма ШВАРЦА. Пусть $\varphi$ - ограниченная голоморфная функиия в единичном шаре $\mathbb{B}^{1} \in \mathbb{C}^{1}$. Тогда для любого $z \in \mathbb{B}^{1}$ выполняется оченка

$$
|\partial \varphi(z)| \leqslant \frac{\|\varphi\|_{H \infty}}{\left(1-|z|^{2}\right)}
$$

Пусть в дальнейшем

$$
r(a, m)=\left(1-\|a\|^{2}+|\langle a, \bar{m}\rangle|^{2}\right)^{1 / 2}
$$

обозначает радиус кругового сечения шара $\mathbb{B}^{n}$ комплексной прямой, проходящей через точку $a$ в направлении $m$.

Из леммы Шварца сразу получаем следующее

СлеДСТвиЕ. 1) Пусть $f \in H^{\infty}\left(\mathbb{D}^{n}\right)$. Тогда для любого $а \in \mathbb{D}^{n}$ и любого $s$, $1 \leqslant s \leqslant n$, будем иметь

$$
\left|\partial_{s} f(a)\right| \leqslant \frac{\|f\|_{H^{\infty}}}{\left(1-\left|a^{s}\right|^{2}\right)}
$$

2) Пусть $f \in H^{\infty}\left(\mathbb{B}^{n}\right)$. Тогда для любого $a \in \mathbb{B}^{n}$ и любого единичного вектора $m \in \mathbb{C}^{n},|m|=1$, справедлива оченка

$$
\left|\partial_{m} f(a)\right|:=\left|\sum_{s=1}^{n} \partial_{s} f(a) m^{s}\right| \leqslant\|f\|_{H^{\infty}} \cdot\left|\frac{r}{r^{2}-|\langle a, \bar{m}\rangle|^{2}}\right|
$$


ДокАЗАТЕльство. Чтобы доказать пункт 1) достаточно заметить, что ограничение $f$ на $s$-ю координатную плоскость в $\mathbb{C}^{n}$ удовлетворяет условиям леммы Шварца. Докажем пункт 2). Для этого обозначим $a^{\prime}=a-\langle a, \bar{m}\rangle$. Тогда функция $g(\zeta):=f\left(a^{\prime}+r \zeta m\right)$ удовлетворяет условиям леммы Шварца. Поэтому

$$
\left|\partial g\left(\frac{\langle a, \bar{m}\rangle}{r}\right)\right| \leqslant\|g\|_{H \infty} \cdot\left|\frac{1}{1-\frac{|\langle a, \bar{m}\rangle|^{2}}{r^{2}}}\right|=\|g\|_{H \infty} \cdot\left|\frac{r^{2}}{r^{2}-|\langle a, \bar{m}\rangle|^{2}}\right| .
$$

С другой стороны,

$$
\begin{aligned}
\partial g\left(\frac{\langle a, \bar{m}\rangle}{r}\right) & =\sum_{s=1}^{n} \frac{\partial f}{\partial z^{s}}\left(a^{\prime}+r \frac{\langle a, \bar{m}\rangle}{r} m\right)\left(\frac{\partial\left(a^{s}+r \zeta m^{s}\right)}{\partial \zeta}\right) \\
& =r \sum_{s=1}^{n} \partial_{s} f(a) m^{s}=r \partial_{m} f(a)
\end{aligned}
$$

откуда и следует утверждение.

Теперь мы можем дать определение интерполяционной последовательности для задачи интерполяции с градиентом в алгебрах $H^{\infty}\left(\mathcal{D}^{n}\right)$.

Зафиксируем последовательность точек $A=\left\{a_{k} \in \mathcal{D}^{n}\right\}_{k=1}^{\infty}$ в области $\mathcal{D}^{n}$. Назовем последовательность комплексных чисел $\left\{f_{k}\right\}_{k=1}^{\infty}$ и векторов $\left\{b_{k} \in \mathbb{C}^{n}\right\}_{k=1}^{\infty} A$-допустuмой для алгебры $H^{\infty}\left(\mathcal{D}^{n}\right)$, если найдутся константы $M_{1}, M_{2}>0$ такие, что

1) $\left|f_{k}\right| \leqslant M_{1}$ для любого $k \in \mathbb{N}$;

2 a) в случае полидиска $\left|b_{k}^{s}\right| \leqslant M_{2} /\left(1-\left|a_{k}^{s}\right|^{2}\right)$ для любых $1 \leqslant s \leqslant n, k \in \mathbb{N}$,

2b) в случае шара

$$
\left|\left\langle b_{k}, m\right\rangle\right|=\left|\sum_{s=1}^{n} b_{k}^{s} m^{s}\right| \leqslant M_{2} \frac{r\left(a_{k}, m\right)}{r\left(a_{k}, m\right)^{2}-\left|\left\langle a_{k}, \bar{m}\right\rangle\right|^{2}}
$$

для любого единичного вектора $m \in \mathbb{C}^{n},|m|=1$, и любого $k \in \mathbb{N}$.

ОПРЕДЕЛЕНИЕ. Последовательность $A=\left\{a_{k}\right\}_{k=1}^{\infty}$ точек $\mathcal{D}^{n}$ называется интерполяиионной для $H^{\infty}\left(\mathcal{D}^{n}\right)$ в задаче интерполяции с градиентом, если для любой $A$-допустимой последовательности чисел $\left\{f_{k}\right\}_{k=1}^{\infty}$ и векторов $\left\{b_{k} \in \mathbb{C}^{n}\right\}_{k=1}^{\infty}$ найдется голо-

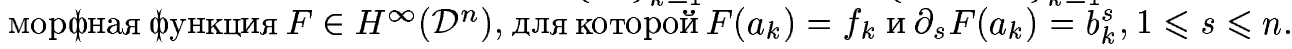

В дальнейшем нам понадобится следующая лемма, доказанная Берндтссоном в [2].

ЛЕмма D. Пусть последовательность точек единичного иара $\left\{a_{k}\right\}_{k=1}^{\infty}$ такова, что существует константа $\delta>0$ такая, что для любого $k \in \mathbb{N}$ справедлива оценка

$$
\prod_{j: j \neq k} \rho\left(a_{j}, a_{k}\right) \geqslant \delta .
$$

Тогда найдется константа $M=M(\delta)>0$ такая, что для любого $z \in \mathbb{B}^{n}$ имеет место неравенство

$$
\sum_{k=1}^{\infty}\left(\frac{1-\left|a_{k}\right|^{2}}{1-\left\langle z, \overline{a_{k}}\right\rangle}\right)^{4}\left|W\left(a_{k}, z\right)\right| \leqslant M(\delta)
$$


где функиия $W(\zeta, z)$ определяется формулой (см. [2])

$$
W(\zeta, z)=\exp \left[-\sum_{j=1}^{\infty}\left(\frac{1+\left\langle z, \overline{a_{j}}\right\rangle}{1-\left\langle z, \overline{a_{j}}\right\rangle}-\frac{1+\left\langle\zeta, \overline{a_{j}}\right\rangle}{1-\left\langle\zeta, \overline{a_{j}}\right\rangle}\right) \cdot\left(\frac{\left(1-\left|a_{j}\right|^{2}\right)\left(1-|\zeta|^{2}\right)}{1-\left|\left\langle\zeta, \overline{a_{j}}\right\rangle\right|^{2}}\right)\right]
$$

$\partial \Omega_{s} \zeta, z \in \mathbb{B}^{n}$.

3. Формулировки и доказательства теорем об интерполяции с градиентом. Аналог теоремы $\mathrm{C}$ из введения для задачи интерполяции с градиентом в полидиске формулируется следуюшим образом.

ТЕОРема 1. Последовательность точек $A=\left\{a_{k}\right\}_{k=1}^{\infty}$ единичного полидиска $\mathbb{D}^{n}$ является интерполяиионной для задачи интерполяиии с градиентом в алгебре $H^{\infty}\left(\mathbb{D}^{n}\right)$, если существует константа $\delta>0$ такая, что для любих $1 \leqslant s \leqslant n u$ $k \in \mathbb{N}$ выполняется неравенство

$$
\prod_{j: j \neq k} \rho^{s}\left(a_{j}, a_{k}\right) \geqslant \delta
$$

Для задачи интерполяции с градиентом в шаре $\mathbb{B}^{n}$ аналог теоремы $\mathrm{B}$ из введения имеет следуюший вид.

Теорема 2. Последовательность точек $A=\left\{a_{k}\right\}_{k=1}^{\infty}$ единичного шара $\mathbb{B}^{n}$ является интерполяиионной для задачи интерполячии с градиентом в алгебре $H^{\infty}\left(\mathbb{B}^{n}\right)$, если выполняются следующие условия:

1) существует константа $\delta_{1}>0$ такая, что

$$
\prod_{j: j \neq k} \rho\left(a_{j}, a_{k}\right) \geqslant \delta_{1}
$$

для любого $k \in \mathbb{N}$

2) найдутся ортонормированный базис $m_{1}, \ldots, m_{n}$ в $\mathbb{C}^{n}$ и константа $\delta_{2}>0$ такие, что

$$
\prod_{j: j \neq k} \rho_{a_{j}, m_{s}}\left(a_{j}, a_{k}\right) \geqslant \delta_{2}
$$

для любых $1 \leqslant s \leqslant n u k \in \mathbb{N}$, где

$$
\rho_{a, m}(z, w)=\left|\frac{r(a, m)(\langle z, \bar{m}\rangle-\langle w, \bar{m}\rangle)}{r(a, m)^{2}-\langle z, \bar{m}\rangle \overline{\langle w, \bar{m}\rangle}}\right|
$$

обозначает гиперболическое расстояние между проекииями точек $z$ u $w$ на комплексную прямую, проходящую через точку а в направлении вектора $m$, a $r(a, m)=\left(1-\|a\|^{2}+|\langle a, \bar{m}\rangle|^{2}\right)^{1 / 2}$ - радиус кругового сечения $\mathbb{B}^{n}$ этой комплексной прямой. 
ДокАЗАТЕльСтво тЕоремы 1 . Пусть дана $A$-допустимая последовательность комплексных чисел $\left\{f_{k}\right\}_{k=1}^{\infty}$ и векторов $\left\{b_{k} \in \mathbb{C}^{n}\right\}_{k=1}^{\infty}$ для алгебры $H^{\infty}\left(\mathbb{D}^{n}\right)$, удовлетворяющая условиям 1$), 2 \mathrm{a}$ ) из определения (п.2). Построим функцию $F \in H^{\infty}\left(\mathbb{D}^{n}\right)$ такую, что $F\left(a_{k}\right)=f_{k}$ и $\partial_{s} F\left(a_{k}\right)=b_{k}^{s}$.

Заметим, прежде всего, что из условия 2) теоремы 1 вытекает условие 1) теоремы С. Следовательно, последовательность $\left\{a_{k} \in \mathbb{D}^{n}\right\}_{k=1}^{\infty}$ является интерполящионной для алгебры $H^{\infty}\left(\mathbb{D}^{n}\right)$, т.е. для заданного набора комплексных чисел $\left\{f_{k}\right\}_{k=1}^{\infty}$ найдется функция $F_{1} \in H^{\infty}\left(\mathbb{D}^{n}\right)$ такая, что $F_{1}\left(a_{k}\right)=f_{k}$. При этом, в силу следствия, сушествует константа $M_{3}>0$, для которой вьполнено неравенство

$$
\left|\partial_{s} F_{1}\left(a_{k}\right)\right| \leqslant \frac{M_{3}}{\left(1-\left|a_{k}\right|^{2}\right)}
$$

для любых $k \in \mathbb{N}$ и $1 \leqslant s \leqslant n$.

Построим голоморфную функцию $G \in H^{\infty}\left(\mathbb{D}^{n}\right)$ такую, что $G\left(a_{k}\right)=0$ и $\partial_{s} G\left(a_{k}\right)=$ $b_{k}^{s}-\partial_{s} F_{1}\left(a_{k}\right)$. Тогда функция $F=F_{1}+G \in H^{\infty}\left(\mathbb{D}^{n}\right)$ будет удовлетворять условиям теоремы 1.

Введем обозначение $c_{k}^{s}:=b_{k}^{s}-\partial_{s} F_{1}\left(a_{k}\right)$ и заметим, что комплексные числа $c_{k}^{s}$ для любых $k \in \mathbb{N}$ и $1 \leqslant s \leqslant n$ удовлетворяют условию

$$
\left|c_{k}^{s}\right| \leqslant \frac{M_{4}}{\left(1-\left|a_{k}\right|^{2}\right)}
$$

с константой $M_{4}=M_{2}+M_{3}$.

$\Phi$ ункцию $G \in H^{\infty}\left(\mathbb{D}^{n}\right)$ будем искать в виде

$$
G=\sum_{k=1}^{\infty} \sum_{s=1}^{n} c_{k}^{s} G_{k s}
$$

где $G_{k s}$ - некоторые функции, удовлетворяющие следующим условиям:

1) для любых $k, l \in \mathbb{N}, 1 \leqslant s \leqslant n$ вьполняется соотношение $G_{k s}\left(a_{l}\right)=0$;

2) для любых $k, l \in \mathbb{N}, 1 \leqslant s, t \leqslant n$ выполняется соотношение $\partial_{t} G_{k s}\left(a_{l}\right)=\delta_{t s} \delta_{k l}$;

3) $G \in H^{\infty}\left(\mathbb{D}^{n}\right)$, т.е. сушествует константа $C>0$ такая, что для любого $z \in \mathbb{D}^{n}$ вьполняется неравенство $\sum_{k=1}^{\infty} \sum_{s=1}^{n}\left|c_{k}^{s}\right| \cdot\left|G_{k s}(z)\right| \leqslant C$.

$\Phi$ ункции $G_{k s}$ построим по следующей формуле

$$
\begin{aligned}
G_{k s}(z)= & \prod_{j: j \neq k}\left[\frac{z^{s}-a_{j}^{s}}{1-z^{s} \overline{a_{j}^{s}}}\right]^{2} \cdot\left[\frac{z^{s}-a_{k}^{s}}{1-z^{s} \overline{a_{k}^{s}}}\right] \\
& \times \prod_{j: j \neq k}\left[\frac{a_{k}^{s}-a_{j}^{s}}{1-a_{k}^{s} \overline{a_{j}^{s}}}\right]^{-2} \cdot\left[1-\left|a_{k}^{s}\right|^{2}\right]\left[\frac{1-\left|a_{k}^{s}\right|^{2}}{1-z^{s} \overline{a_{k}^{s}}}\right]^{4} \cdot W\left(a_{k}^{s}, z^{s}\right),
\end{aligned}
$$

где функции $W$ задаются формулой из леммы $\mathrm{D}$ при $n=1$

$$
W(\zeta, z)=\exp \left[-\sum_{j=1}^{\infty}\left(\frac{1+\left\langle z, \overline{a_{j}}\right\rangle}{1-\left\langle z, \overline{a_{j}}\right\rangle}-\frac{1+\left\langle\zeta, \overline{a_{j}}\right\rangle}{1-\left\langle\zeta, \overline{a_{j}}\right\rangle}\right)\left(\frac{\left(1-\left|a_{j}\right|^{2}\right)\left(1-|\zeta|^{2}\right)}{1-\left|\left\langle\zeta, \overline{a_{j}}\right\rangle\right|^{2}}\right)\right] .
$$

Формулы для $G_{k s}$ определены корректно, так как все бесконечные произведения и суммы сходятся в силу условия 2) из теоремы 1 и леммы $\mathrm{D}$. 
Покажем, что построенные функции $G_{k s}$ обладают приведенными вьше свойствами 1)-3).

Действительно, функции $G_{k s}$ представляются в виде

$$
G_{k s}(z)=\varphi(z)\left(z^{s}-a_{l}^{s}\right),
$$

где $\varphi$ - некоторая голоморфная функция. Отсюда следует свойство 1$): G_{k s}\left(a_{l}\right)=0$ для любых $k, l \in \mathbb{N}$ и $1 \leqslant s \leqslant n$.

Для проверки свойства 2) заметим, что при $t \neq s$ функция $G_{k s}(z)$ не зависит от $z^{t}$, откуда следует, что $\partial_{t} G_{k s}\left(a_{l}\right)=0$ при $t \neq s$. Поскольку функция $G_{k s}$ представима в виде

$$
G_{k s}(z)=\psi\left(z^{s}\right)\left(z^{s}-a_{k}^{s}\right)
$$

для некоторой голоморфной функции $\psi$, удовлетворяющей $\psi\left(a_{k}^{s}\right)=1$, то $\partial_{s} G_{k s}\left(a_{k}\right)=1$, т.е. свойство 2) выполняется при $t=s, k=l$. Наконец, из представления

$$
G_{k s}(z)=\omega\left(z^{s}\right)\left(z^{s}-a_{l}^{s}\right)^{2},
$$

выполненного для некоторой голоморфной функции $\omega$ при $l \neq k$, следует, что $\partial_{s}\left(G_{k s}\right)\left(a_{l}\right)=0$; тем самым, свойство 2) справедливо и при $t=s, k \neq l$.

Перейдем к доказательству свойства 3$)$. Для любого $z \in \mathbb{D}^{n}$ справедливо неравенство

$$
\begin{gathered}
\sum_{k=1}^{\infty} \sum_{s=1}^{n}\left|c_{k}^{s}\right| \cdot\left|G_{k s}(z)\right| \leqslant \sum_{k=1}^{\infty} \sum_{s=1}^{n}\left|c_{k}^{s}\right| \prod_{j: j \neq k}\left|\frac{z^{s}-a_{j}^{s}}{1-z^{s} \overline{a_{j}^{s}}}\right|^{2} \cdot\left|\frac{z^{s}-a_{k}^{s}}{1-z^{s} \overline{a_{k}^{s}}}\right| \\
\quad \times\left.\left.\prod_{j: j \neq k}\left|\frac{a_{k}^{s}-a_{j}^{s}}{1-a_{k}^{s} \overline{a_{j}^{s}}}\right|^{-2} \cdot|1-| a_{k}^{s}\right|^{2}|\cdot| \frac{1-\left|a_{k}^{s}\right|^{2}}{1-z^{s} \overline{a_{k}^{s}}}\right|^{4} \cdot\left|W\left(a_{k}^{s}, z^{s}\right)\right| .
\end{gathered}
$$

Tak как

$$
\prod_{j: j \neq k}\left|\frac{z^{s}-a_{j}^{s}}{1-z^{s} \overline{a_{j}^{s}}}\right|^{2} \cdot\left|\frac{z^{s}-a_{k}^{s}}{1-z^{s} \overline{a_{k}^{s}}}\right| \leqslant 1
$$

для любого $z \in \mathbb{D}^{n}$, правая часть неравенства (5) оценивается через

$$
\left.\left.\sum_{k=1}^{\infty} \sum_{s=1}^{n}\left|c_{k}^{s}\right| \prod_{j: j \neq k}\left|\frac{a_{k}^{s}-a_{j}^{s}}{1-a_{k}^{s} \overline{a_{j}^{s}}}\right|^{-2} \cdot|1-| a_{k}^{s}\right|^{2}|\cdot| \frac{1-\left|a_{k}^{s}\right|^{2}}{1-z^{s} \overline{a_{k}^{s}}}\right|^{4} \cdot\left|W\left(a_{k}^{s}, z^{s}\right)\right| .
$$

По условию теоремы

$$
\prod_{j: j \neq k}\left|\frac{a_{k}^{s}-a_{j}^{s}}{1-a_{k}^{s} \overline{a_{j}^{s}}}\right|^{-2} \leqslant \delta^{-2}
$$

поэтому выражение (6) можно оценить через

$$
\left.\left.\delta^{-2} \sum_{k=1}^{\infty} \sum_{s=1}^{n}\left|c_{k}^{s}\right||1-| a_{k}^{s}\right|^{2}|\cdot| \frac{1-\left|a_{k}^{s}\right|^{2}}{1-z^{s} \overline{a_{k}^{s}}}\right|^{4} \cdot\left|W\left(a_{k}^{s}, z^{s}\right)\right|
$$

Поскольку $\left.\left|c_{k}^{s}\right||1-| a_{k}^{s}\right|^{2} \mid \leqslant M_{4}$, последнее выражение не превосходит

$$
\delta^{-2} M_{4} \sum_{k=1}^{\infty} \sum_{s=1}^{n}\left|\frac{1-\left|a_{k}^{s}\right|^{2}}{1-z^{s} \overline{a_{k}^{s}}}\right|^{4} \cdot\left|W\left(a_{k}^{s}, z^{s}\right)\right| .
$$


Пользуясь леммой D, мы можем оценить (7) выражением вида

$$
\delta^{-2} M_{4} \sum_{s=1}^{n} M(\delta) \leqslant \text { const } .
$$

Окончательно,

$$
\sum_{k=1}^{\infty} \sum_{s=1}^{n}\left|c_{k}^{s}\right| \cdot\left|G_{k s}(z)\right| \leqslant \text { const } .
$$

Тем самым показано, что функции $G_{k s}, G, F$ удовлетворяют требуемым условиям, откуда вытекает утверждение теоремы 1.

Теорема 2 доказывается с помошью аналогичных рассуждений.

ДокАЗАТЕЛЬСтво тЕоремы 2. Пусть дана $A$-допустимая последовательность комплексных чисел $\left\{f_{k}\right\}_{k=1}^{\infty}$ и векторов $\left\{b_{k} \in \mathbb{C}^{n}\right\}_{k=1}^{\infty}$ для алгебры $H^{\infty}\left(\mathbb{B}^{n}\right)$, удовлетворяющая условиям 1$), 2 \mathrm{~b}$ ) из определения (п. 2). Построим функцию $F \in H^{\infty}\left(\mathbb{B}^{n}\right)$ такую, что $F\left(a_{k}\right)=f_{k}$ и $\partial_{s} F\left(a_{k}\right)=b_{k}^{s}$.

Заметим снова, что из условия 3$)$ теоремы 2 следует в силу теоремы В, что последовательность $\left\{a_{k} \in \mathbb{B}^{n}\right\}_{k=1}^{\infty}$ является интерполящионной для алгебры $H^{\infty}\left(\mathbb{B}^{n}\right)$, т.е. для заданного набора комплексных чисел $\left\{f_{k}\right\}_{k=1}^{\infty}$ найдется функция $F_{1} \in H^{\infty}\left(\mathbb{B}^{n}\right)$ такая, что $F_{1}\left(a_{k}\right)=f_{k}$. Далее, в силу следствия сушествует константа $M_{3}$, для которой выполнено неравенство

$$
\left|\partial_{m_{s}} F_{1}\left(a_{k}\right)\right|=\left|\sum_{t=1}^{n} \partial_{t} F_{1}\left(a_{k}\right) m_{s}^{t}\right| \leqslant M_{3} \frac{r\left(a_{k}, m_{s}\right)}{\left(r^{2}\left(a_{k}, m_{s}\right)-\left|\left\langle a_{k}, \overline{m_{s}}\right\rangle\right|^{2}\right)}
$$

для любых $k \in \mathbb{N}$ и $1 \leqslant s \leqslant n$.

Построим функцию $G \in H^{\infty}\left(\mathbb{B}^{n}\right)$ такую, что $G\left(a_{k}\right)=0$ и $\partial_{s} G\left(a_{k}\right)=b_{k}^{s}-\partial_{s} F_{1}\left(a_{k}\right)$. Тогда функция $F=F_{1}+G \in H^{\infty}\left(\mathbb{B}^{n}\right)$ будет удовлетворять условиям теоремы 2 .

Введем обозначение $c_{k}^{s}:=b_{k}^{s}-\partial_{s} F_{1}\left(a_{k}\right)$ и заметим, что комплексные числа $c_{k}^{s}$ для любых $k \in \mathbb{N}$ и $1 \leqslant s \leqslant n$ удовлетворяют условию

$$
\left|\sum_{t=1}^{n} c_{k}^{s} m_{s}^{t}\right| \leqslant M_{4} \frac{r\left(a_{k}, m_{s}\right)}{\left(r^{2}\left(a_{k}, m_{s}\right)-\left|\left\langle a_{k}, \overline{m_{s}}\right\rangle\right|^{2}\right)}
$$

с константой $M_{4}=M_{2}+M_{3}$.

$\Phi$ ункцию $G \in H^{\infty}\left(\mathbb{B}^{n}\right)$ будем искать в виде

$$
G=\sum_{k=1}^{\infty} \sum_{s=1}^{n} d_{k s} G_{k s}
$$

г де $d_{k s}$ - некоторые комплексные числа, a $G_{k s}$ - функции, удовлетворяющие следующим условиям :

1) для любых $k, l \in \mathbb{N}, 1 \leqslant s \leqslant n$ вьполняется соотношение $G_{k s}\left(a_{l}\right)=0$;

2) для любых $k, l \in \mathbb{N}, 1 \leqslant s, t \leqslant n$ вьполняются соотношения $\partial_{t} G_{k s}\left(a_{l}\right)=\overline{m_{s}^{t}} \delta_{k l}$ и $\sum_{s=1}^{n} d_{k s} m_{s}=c_{k}$, т.е. $d_{k s}=\sum_{t=1}^{n} c_{k}^{t} m_{s}^{t}$ в силу ортонормированности базиса $\left\{m_{s}\right\}$

3) $G \in H^{\infty}\left(\mathbb{B}^{n}\right)$, т.е. существует константа $C>0$ такая, что для любого $z \in \mathbb{B}^{n}$ вьполняется неравенство $\sum_{k=1}^{\infty} \sum_{s=1}^{n}\left|d_{k s}\right|\left|G_{k s}(z)\right| \leqslant C$. 
$\Phi$ ункции $G_{k s}$ построим по следующей формуле

$$
\begin{aligned}
G_{k s}(z)= & \prod_{j: j \neq k}\left[\frac{r\left(a_{j}, m_{s}\right)\left(\left\langle z, \overline{m_{s}}\right\rangle-\left\langle a_{j}, \overline{m_{s}}\right\rangle\right)}{r^{2}\left(a_{j}, m_{s}\right)-\left\langle z, \overline{m_{s}}\right\rangle \overline{\left\langle a_{j}, \overline{m_{s}}\right\rangle}}\right]^{2} \\
& \times\left[\frac{r\left(a_{k}, m_{s}\right)\left(\left\langle z, \overline{m_{s}}\right\rangle-\left\langle a_{k}, \overline{m_{s}}\right\rangle\right)}{r^{2}\left(a_{k}, m_{s}\right)-\left\langle z, \overline{m_{s}}\right\rangle \overline{\left\langle a_{k}, \overline{m_{s}}\right\rangle}}\right] \\
& \times \prod_{j: j \neq k}\left[\frac{r\left(a_{j}, m_{s}\right)\left(\left\langle a_{k}, \overline{m_{s}}\right\rangle-\left\langle a_{j}, \overline{m_{s}}\right\rangle\right)}{r^{2}\left(a_{j}, m_{s}\right)-\left\langle a_{k}, \overline{m_{s}}\right\rangle \overline{\left\langle a_{j}, \overline{m_{s}}\right\rangle}}\right]^{-2} \cdot \frac{r^{2}\left(a_{k}, m_{s}\right)-\left|\left\langle a_{k}, \overline{m_{s}}\right\rangle\right|^{2}}{r\left(a_{k}, m_{s}\right)} \\
& \times\left[\frac{1-\left|a_{k}^{s}\right|^{2}}{1-z^{s} \overline{a_{k}^{s}}}\right]^{4} \cdot W\left(a_{k}, z\right),
\end{aligned}
$$

где

$$
W(\zeta, z)=\exp \left[-\sum_{j=1}^{\infty}\left(\frac{1+\left\langle z, \overline{a_{j}}\right\rangle}{1-\left\langle z, \overline{a_{j}}\right\rangle}-\frac{1+\left\langle\zeta, \overline{a_{j}}\right\rangle}{1-\left\langle\zeta, \overline{a_{j}}\right\rangle}\right) \cdot\left(\frac{\left(1-\left|a_{j}\right|^{2}\right)\left(1-|\zeta|^{2}\right)}{1-\left|\left\langle\zeta, \overline{a_{j}}\right\rangle\right|^{2}}\right)\right]
$$

- функции из леммы D.

Формулы для $G_{k s}$ определены корректно, так как все бесконечные произведения и суммы сходятся в силу условий 3$), 4)$ из теоремы 2 и леммы D.

Покажем, что построенные функции $G_{k s}$ обладают приведенными вьше свойствами 1), 2), 3).

Действительно, для любого $l$ функции $G_{k s}$ можно представить в виде

$$
G_{k s}(z)=\varphi(z)\left\langle\left(z-a_{l}\right), \overline{m_{s}}\right\rangle,
$$

где $\varphi$ - некоторая голоморфная функция. Отсюда следует свойство 1$): G_{k s}\left(a_{l}\right)=0$ для любых $k, l \in \mathbb{N}$ и $1 \leqslant s \leqslant n$.

Для проверки свойства 2 ) заметим, что поскольку функция $G_{k s}$ представима в виде

$$
G_{k s}(z)=\psi(z)\left\langle\left(z-a_{k}\right), \overline{m_{s}}\right\rangle
$$

для некоторой голоморфной функции $\psi$, удовлетворяющей $\psi\left(a_{k}^{s}\right)=1$, то $\partial_{t} G_{k s}\left(a_{k}\right)=$ $\overline{m_{s}^{t}}$, т.е. свойство 2) выполняется при $k=l$. Наконец, из представления

$$
G_{k s}(z)=\omega(z)\left\langle\left(z-a_{l}\right), \overline{m_{s}}\right\rangle^{2},
$$

выполненного для некоторой голоморфной функции $\omega$ при $l \neq k$, следует, что $\partial_{t}\left(G_{k s}\left(a_{l}\right)\right)=0$; тем самым, свойство 2) справедливо и при $k \neq l$.

Перейдем к доказательству свойства 3$)$. Для любого $z \in \mathbb{B}^{n}$ справедливо неравенство

$$
\begin{aligned}
\sum_{k=1}^{\infty} \sum_{s=1}^{n}\left|d_{k s}\right|\left|G_{k s}(z)\right| \leqslant & \sum_{k=1}^{\infty} \sum_{s=1}^{n}\left|d_{k s}\right| \prod_{j: j \neq k} \mid \frac{r\left(a_{j}, m_{s}\right)\left(\left\langle z, \overline{m_{s}}\right\rangle-\left\langle a_{j}, \overline{m_{s}}\right\rangle\right)}{r^{2}\left(a_{j}, m_{s}\right)-\left.\left\langle z, \overline{m_{s}}\right\rangle \overline{\left\langle a_{j}, \overline{m_{s}}\right\rangle}\right|^{2}} \\
& \times \mid \frac{r\left(a_{k}, m_{s}\right)\left(\left\langle z, \overline{m_{s}}\right\rangle-\left\langle a_{k}, \overline{m_{s}}\right\rangle\right)}{r^{2}\left(a_{k}, m_{s}\right)-\left\langle z, \overline{m_{s}}\right\rangle \overline{\left\langle a_{k}, \overline{m_{s}}\right\rangle} \mid} \\
& \times \prod_{j: j \neq k} \mid \frac{r\left(a_{j}, m_{s}\right)\left(\left\langle a_{k}, \overline{m_{s}}\right\rangle-\left\langle a_{j}, \overline{m_{s}}\right\rangle\right)}{r^{2}\left(a_{j}, m_{s}\right)-\left.\left\langle a_{k}, \overline{m_{s}}\right\rangle \overline{\left\langle a_{j}, \overline{m_{s}}\right\rangle}\right|^{-2}} \\
& \times\left|\frac{r^{2}\left(a_{k}, m_{s}\right)-\left|\left\langle a_{k}, \overline{m_{s}}\right\rangle\right|^{2}}{r\left(a_{k}, m_{s}\right)}\right|\left|\frac{1-\left|a_{k}^{s}\right|^{2}}{1-z^{s} \overline{a_{k}^{s}}}\right|^{4} \cdot\left|W\left(a_{k}^{s}, z^{s}\right)\right| .
\end{aligned}
$$


Так как для любого $z \in \mathbb{B}^{n}$

$$
\prod_{j: j \neq k}\left|\frac{r\left(a_{j}, m_{s}\right)\left(\left\langle z, \overline{m_{s}}\right\rangle-\left\langle a_{j}, \overline{m_{s}}\right\rangle\right)}{r^{2}\left(a_{j}, m_{s}\right)-\left\langle z, \overline{m_{s}}\right\rangle \overline{\left\langle a_{j}, \overline{m_{s}}\right\rangle}}\right|^{2}\left|\frac{r\left(a_{k}, m_{s}\right)\left(\left\langle z, \overline{m_{s}}\right\rangle-\left\langle a_{k}, \overline{m_{s}}\right\rangle\right)}{r^{2}\left(a_{k}, m_{s}\right)-\left\langle z, \overline{m_{s}}\right\rangle \overline{\left\langle a_{k}, \overline{m_{s}}\right\rangle}}\right| \leqslant 1,
$$

из этого неравенства получаем следующую оценку на правую часть (8)

$$
\begin{aligned}
& \sum_{k=1}^{\infty} \sum_{s=1}^{n}\left|d_{k s}\right| \prod_{j: j \neq k}\left|\frac{r\left(a_{j}, m_{s}\right)\left(\left\langle a_{k}, \overline{m_{s}}\right\rangle-\left\langle a_{j}, \overline{m_{s}}\right\rangle\right)}{r^{2}\left(a_{j}, m_{s}\right)-\left\langle a_{k}, \overline{m_{s}}\right\rangle \overline{\left\langle a_{j}, \overline{m_{s}}\right\rangle}}\right|^{-2} \\
& \times\left|\frac{r^{2}\left(a_{k}, m_{s}\right)-\left|\left\langle a_{k}, \overline{m_{s}}\right\rangle\right|^{2}}{r\left(a_{k}, m_{s}\right)}\right| \cdot\left|\frac{1-\left|a_{k}^{s}\right|^{2}}{1-z^{s} \overline{a_{k}^{s}}}\right|^{4} \cdot\left|W\left(a_{k}^{s}, z^{s}\right)\right| .
\end{aligned}
$$

В силу того, что

$$
\prod_{j: j \neq k}\left|\frac{r\left(a_{j}, m_{s}\right)\left(\left\langle a_{k}, \overline{m_{s}}\right\rangle-\left\langle a_{j}, \overline{m_{s}}\right\rangle\right)}{r^{2}\left(a_{j}, m_{s}\right)-\left\langle a_{k}, \overline{m_{s}}\right\rangle \overline{\left\langle a_{j}, \overline{m_{s}}\right\rangle}}\right|^{-2} \leqslant \delta_{2}^{-2}
$$

по условию (4) выражение (9) можно оценить через

$$
\delta_{2}^{-2} \sum_{k=1}^{\infty} \sum_{s=1}^{n}\left|d_{k s}\right|\left|\frac{r^{2}\left(a_{k}, m_{s}\right)-\left|\left\langle a_{k}, \overline{m_{s}}\right\rangle\right|^{2}}{r\left(a_{k}, m_{s}\right)}\right| \cdot\left|\frac{1-\left|a_{k}^{s}\right|^{2}}{1-z^{s} \overline{a_{k}^{s}}}\right|^{4} \cdot\left|W\left(a_{k}^{s}, z^{s}\right)\right| .
$$

Применяя оценку

$$
\begin{aligned}
& \sum_{s=1}^{n}\left|d_{k s}\right|\left|\frac{r^{2}\left(a_{k}, m_{s}\right)-\left|\left\langle a_{k}, \overline{m_{s}}\right\rangle\right|^{2}}{r\left(a_{k}, m_{s}\right)}\right| \\
& \quad=\sum_{s=1}^{n}\left|\sum_{t=1}^{n} c_{k}^{t} m_{s}^{t}\right|\left|\frac{r^{2}\left(a_{k}, m_{s}\right)-\left|\left\langle a_{k}, \overline{m_{s}}\right\rangle\right|^{2}}{r\left(a_{k}, m_{s}\right)}\right| \leqslant \sum_{s=1}^{n} M_{4} \leqslant n M_{4},
\end{aligned}
$$

получим для выражения (10) оценку вида

$$
\delta_{2}^{-2} n M_{4} \sum_{k=1}^{\infty}\left|\frac{1-\left|a_{k}^{s}\right|^{2}}{1-z^{s} \overline{a_{k}^{s}}}\right|^{4} \cdot\left|W\left(a_{k}^{s}, z^{s}\right)\right| \leqslant \text { const }
$$

(последнее неравенство вытекает из леммы D).

Резюмируя, получаем

$$
\sum_{k=1}^{\infty} \sum_{s=1}^{n}\left|d_{k s}\right|\left|G_{k s}(z)\right| \leqslant \text { const. }
$$

Тем самым показано, что функции $G_{k s}, G, F$ удовлетворяют требуемым условиям, откуда вытекает утверждение теоремы 2.

\section{СПИСОК ЦИТИРОВАННОЙ ЛИТЕРАТУРЫ}

[1] Carleson L. An interpolation problem for bounded analytic functions // Amer. J. Math. 1958. V. 8. P. 921-930.

[2] Berndtsson B. Interpolating sequences for $H^{\infty}$ in the ball // Indag. Math. 1985. V. 88. P. 1-10.

[3] Berndtsson B. Interpolating sequences in the polydisk // Trans. Amer. Math. Soc. 1987. V. 302. P. 161-169.

[4] Varopoulos N. Th. Sur une probléme d'interpolation // C. R. Acad. Sci. Paris. Sér. A. 1972. V. 274. P. 1539-1542. 